Originalien

Med Klin Intensivmed Notfmed 2021 · 116:687-693 https://doi.org/10.1007/s00063-020-00729-5 Eingegangen: 8. Mai 2020

Überarbeitet: 5. Juli 2020

Angenommen: 4. August 2020

Online publiziert: 2. Oktober 2020

(c) Der/die Autor(en) 2020

\section{Redaktion}

M. Buerke

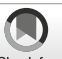

H. M. Orth ${ }^{1,2} \cdot$ S. Al Agha $\cdot$ M. Kempe ${ }^{2,4} \cdot$ C. Mackenzie ${ }^{2,5} \cdot$ M. Michael $^{3} \cdot$

M. Bernhard ${ }^{3} \cdot$ B. -E. O. Jensen ${ }^{1,2}$

${ }^{1}$ Klinik für Gastroenterologie, Hepatologie und Infektiologie, Universitätsklinikum Düsseldorf, Düsseldorf, Deutschland

${ }^{2}$ Antibiotic Stewardship (ABS) Team, Universitätsklinikum Düsseldorf, Düsseldorf, Deutschland

${ }^{3}$ Zentrale Notaufnahme, Universitätsklinikum Düsseldorf, Düsseldorf, Deutschland

${ }^{4}$ Zentralapotheke, Universitätsklinikum Düsseldorf, Düsseldorf, Deutschland

${ }^{5}$ Institiut für Medizinische Mikrobiologie und Krankenhaushygiene, Universitätsklinikum Düsseldorf, Düsseldorf, Deutschland

\title{
Optimierung der mikrobiellen Diagnostik durch Einführung einer Standard Operating Procedure „Blutkulturen“ in der zentralen Notaufnahme
}

Notaufnahme gewonnen werden [6]. In den vergangenen Jahren wiesen Studien aber immer wieder eine schlechte Qualität und Quantität der Blutkulturdiagnostik in Notaufnahmen nach [3, 4]. Falschpositive Befunde durch mit Hautkeimen kontaminierte Blutkulturen können mit einer nichtindizierten Verwendung von Antibiotika einhergehen und die Krankenhauskosten erhöhen [8].

Vor diesem Hintergrund ist es Aufgabe des Antibiotic-Stewardship(ABS)Teams eines Krankenhauses, zusammen mit dem Team aus der zentralen Notaufnahme einen Maßnahmenkatalog zur Verbesserung der Qualität und Quantität der Blutkulturdiagnostik $\mathrm{zu}$ erarbeiten und umzusetzen. Nach Bool et al. [3] sollen entsprechende Maßnahmen 1) Schulungen und Feedback („education and feedback“), 2) die Etablierung von Maßnahmenbündeln/ Standard Operating Procedures (SOP, "bundled approaches") und 3) Technik und Material („technique and equipment") enthalten. Eine Optimierung der Qualität und Quantität der Blutkulturdiagnostik stellt damit einen wesentlichen Beitrag zur Qualitätssteigerung der mikrobiellen Diagnostik und damit auch der antiinfektiven Therapie dar.
Ziel der vorliegenden retrospektiven Untersuchung war es daher zu prüfen, inwieweit die Entwicklung, Einführung und Schulung einer SOP „Blutkulturen“ in einer zentralen Notaufnahme die Qualitäts- und Quantitätsindikatoren für die Blutkulturdiagnostik verbessern können.

\section{Material und Methodik}

Im Rahmen des vorliegenden Projektes wurden die folgenden 5 Punkte durchgeführt:

1. Entwicklung einer SOP „Blutkulturen" durch das ABS-Team und die zentrale Notaufnahme;

2. Einführung eines Blutentnahmeadapters mit Blutkulturhalter in der zentralen Notaufnahme;

3. Schulung in der Anwendung der SOP „Blutkulturen“ und der fachgerechten Abnahme von Blutkulturen in der zentralen Notaufnahme;

4. retrospektiver Vergleich von Prozessindikatoren der Blutkulturdiagnostik in der zentralen Notaufnahme;

5. Punktprävalenzanalyse in der zentralen Notaufnahme.

Die retrospektive Studie wurde durch die Ethikkommission der Heinrich-Heineantibiotischen Therapie unter antiseptischen Bedingungen noch in der zentralen 


\section{1) Indikation:}

- Bei klinischen Zeichen einer Sepsis (klinisch oder laborchemisch) oder Fieber und stationärer Therapie

- anderweitiger Verdacht auf eine schwere Infektion

- V.a. Bakteriämie oder Fungämie

- Katheterassoziierte Infektion

- systemische Beteiligung einer lokalisierten Infektion v.a. bei immunsupprimierten Patienten

\section{2) Zeitpunkt:}

- BK nach Indikationsprüfung so schnell wie möglich vor Antibiotikagabe abnehmen

- im septischen Schock sollte die Antibiotikagabe aber nicht durch mehrmalige Punktionsversuche verzögert werden

- bei laufender Antibiotikatherapie sollten BK möglichst kurz vor der nächsten geplanten Gabe abgenommen werden.
3) Anzahl:

Ein Paar BK bezeichnet

immer eine anaerobe und aerobe BK-Flasche

mind. 2 Paar BK, wenn

möglich aus verschiedenen peripheren Punktionen

3 Paar BK erhöht die Sensitivität bei Sepsis auf $95-98 \%$

- >4 Paar bei infektiöser

Endokarditis (s. SOP

infektiöse Endokarditis)

\section{4) Abnahme-/Bearbeitungsprozess:}

- nicht aus liegenden Kathetern abnehmen und möglichst neue periphere Venenpunktion vornehmen

- Abnahme aus neu angelegtem arteriellem Zugang bzw. zentralen Venenzugang ist möglich

Ausnahme: Katheterassoziierter Sepsis (Abnahme aus liegendem Katheter plus periphere BK)

- hygienische Maßnahmen

-Händedesinfektion vor Anziehen der Handschuhe, immer Schutzhandschuhe zum Eigenschutz

-2-malige Desinfektion, sterile Bedingungen mit Hautdesinfektion und Einhaltung der Einwirkzeit

-Punktion im desinfizierten Bereich, zur Repalpation $\rightarrow$ sterile Handschuhe anlegen

- Punktion

-bevorzugt mit Vacutainer (Abnahmemenge hier durch BK-Flaschen reguliert)

-Punktion im desinfiziertem Bereich nach ausreichenden 0.g. hygienischen Maßnahmen

- Beimpfen der BK-Flaschen

- Entfernen der Plastikkappen und Desinfektion der Membran. Einwirkzeit und Abtrocknung abwarten ( $30 \mathrm{~s}$ )

- BK-Flaschen nicht durch Desinfektionssee beimpfen

- wenn Abnahme mit Spritzen: nicht auf unsterilen Flächen ablegen

- Kultur beimpfen mit 8-10 ml Blut/BK-Flasche (aerob und anaerob)

- kein Wechsel der Kanüle erforderlich, Flaschen nicht belüften

- IMMER auf Dokumentation der BK-Abnahme in COPRA achten

5) Kennzeichnung und Transport:

- Je BK-Flasche ein mikrobiologischer Einsendeschein. Scheinetikett und kleines Namensetikett parallel zum Barcode. Barcode und QR-Code nicht überkleben

- Angabe der Entnahmestelle, der Entnahmezeit, der Indikation und ggf. der laufenden antibiotischen Therapien

- Transport mittels AWT bei Raumtemperatur bis 18.00 Uhr, nach 18.00 Uhr Lagerung vor AWT-Anlage

Abb. $1<$ Standard Operating Procedures (SOP) "Blutkulturen" (BK) mit Darstellung von Indikation, Zeitpunkt, Anzahl, Durchführung, Kennzeichnung und Transport in der zentralen Notaufnahme. (Mit freundlicher Genehmigung, $\odot$ PD Dr. M. Bernhard, alle Rechte vorbehalten)
Universität (Studiennummer: 2019-392-

Retro DEuA) genehmigt.

\section{SOP „Blutkulturen”}

Durch das ABS-Team des Universitätsklinikums Düsseldorf wurde in Zusammenarbeit mit Mitarbeiterinnen und Mitarbeitern der zentralen Notaufnahme ein Konzept zur Optimierung der Quantität und Qualität in Form einer SOP „Blutkulturen" entwickelt (• Abb. 1). Bekannte Vorgaben für die Abnahme von Blutkulturen wurden berücksichtigt und in die SOP „Blutkulturen“ integriert $[1-4,8]$.

\section{Schulungsmaßnahmen}

Durch die Schulungsmaßnahmen des pflegerischen und ärztlichen Personals der Zentralen Notaufnahme des Universitätsklinikums Düsseldorf sollte durch das ABS-Team die Indikationsstellung zur Blutkulturdiagnostik, die Durchführung der Blutkulturabnahme und die weitere Präanalytik standardisiert vermittelt werden. Hierzu erfolgte ein mündlicher Vortrag mit der Dauer von rund $15 \mathrm{~min}$ und einer Gruppengröße von etwa 5 Personen in der zentralen Notaufnahme. Die Anwesenheit wurde mittels Listen erfasst. Der Inhalt der Schulung umfasste die Erläuterung der Bedeutung der Blutkulturdiagnostik, die
Aushändigung und Erläuterung der zugrunde liegenden SOP „Blutkulturen“ sowie die Demonstration der Durchführung der Abnahme von Blutkulturen an einem Dummy. Abgerundet wurden die Schulungsmaßnahmen durch die Möglichkeit, Fragen zu stellen und Unklarheiten $\mathrm{zu}$ beseitigen.

\section{Blutabnahmeadapter}

Vor Umsetzung des Maßnahmenbündels waren Blutkulturen mit einer Standard$20 \mathrm{ml}$-Spritze mit folgender Injektion in 2 Blutkulturflaschen abgenommen worden. Gleichzeitig mit den Schulungsmaßnahmen wurde dann durch die Einführung eines neuen Blutentnahme- 
Med Klin Intensivmed Notfmed 2021 • 116:687-693 https://doi.org/10.1007/s00063-020-00729-5

(c) Der/die Autor(en) 2020

\section{H. M. Orth · S. Al Agha · M. Kempe · C. Mackenzie · M. Michael · M. Bernhard · B. -. O. Jensen}

\section{Optimierung der mikrobiellen Diagnostik durch Einführung einer Standard Operating Procedure „Blutkulturen“ in der zentralen Notaufnahme}

\section{Zusammenfassung}

Einleitung. Zentrale Notaufnahmen stellen die Eintrittspforte für viele stationär aufzunehmende Patienten in einem Krankenhaus dar und sind häufig der Ausgangspunkt für die antiinfektive Diagnostik und Therapie von Notfallpatienten. In dieser retrospektiven Untersuchung soll der Frage nachgegangen werden, wie die Etablierung einer Standard Operating Procedure (SOP) „Blutkulturen“ und deren Schulung die mikrobielle Diagnostik in einer zentralen Notaufnahme verbessern kann.

Methodik. In einer Vorher-und-nachherUntersuchung wurde über einen jeweils 3-monatigen Zeitraum (11/2017 bis $01 / 2018$ und $11 / 2018$ bis $01 / 2019$ ) die Anzahl der abgenommenen Blutkulturen, die Rate an Blutkulturen/1000 Fälle, die Anzahl positiver Blutkulturen und die Häufigkeit typischer Hautkeime analysiert. Im Zeitraum zwischen den evaluierten Zeitabschnitten wurde eine SOP "Blutkulturen“ in Zusammenarbeit mit dem Antibiotic-Stewardship(ABS)-Teams und der zentrale Notaufnahme entwickelt, implementiert und geschult. Ein positives Votum der Ethikkommission der HeinrichHeine-Universität (2019-392-RetroDEuA) lag vor.

Ergebnisse. Die pflegerischen und ärztlichen Mitarbeiter wurden zu $92 \%$ bzw. $93 \%$ geschult. Die Anzahl der abgenommenen Blutkulturen stieg von 1757 auf 2872 um
$64 \%$ ebenso wie die Anzahl der Blutkulturen/1000 Fälle von 287 auf 481 (68\%). Die Anzahl der positiven Blutkulturen reduzierte sich von 18,6 auf $13,7 \%(p<0,05)$. Typische Hautkeime fanden sich in $34,4 \%$ und $26,4 \%$ der Fälle $(p<0,05)$.

Interpretation. Die durch Schulungen begleitete Einführung einer SOP „Blutkulturen“ in der zentralen Notaufnahme kann einen relevanten Beitrag zur antimikrobiellen Diagnostik leisten und sowohl die Quantität als auch die Qualität erhöhen.

Schlüsselwörter

Sepsis · Infektion · Mikrobielle Diagnostik . Antiinfektive Therapie · Blutkulturen

\section{Optimization of microbial diagnostics by introduction of a blood culture standard operating procedure in the emergency department}

\section{Abstract}

Introduction. The emergency department $(E D)$ is the main port of entry for patients with infectious diseases, the place where a number of diagnostic procedures are performed and treatment is often initiated. The aim of this retrospective study was to estimate the influence of the establishment and introduction of a blood culture standard operating procedure (BC-SOP) and of the subsequent training of microbial diagnostics in an ED.

Methods. In a before and after study over a study period of 3 months each (November 2017-January 2018 and November 2018-January 2019), the number of blood cultures taken, the rate of blood cultures per
1000 patients, the number of positive blood cultures and the frequency of typical skin pathogens were evaluated. In the interim time between the two study periods, a BCSOP was developed in collaboration with the hospital's antibiotic stewardship team and subsequently introduced with staff training in the ED. The study was approved by the local ethics committee of the medical faculty of the Heinrich Heine University (2019-392RetroDEuA).

Results. In total $92 \%$ of the nursing personnel and $93 \%$ of the medical personnel received training. The total number of blood cultures increased from 1757 to 2872 (64\% increase) and the rate of blood cultures per $1000 \mathrm{pa}-$ tients from 287 to 481 (68\% increase). The number of positive blood cultures decreased from $18.6 \%$ to $13.7 \%(p<0.05)$. Typical skin pathogens were found in $34.4 \%$ and $26.4 \%$ of the cases, respectively $(p<0.05)$.

Conclusion. The development, introduction and training of a BC-SOP in the ED can make a relevant contribution to the microbial diagnostics and increase the quantity as well as the quality.

\section{Keywords}

Infection · Sepsis - Microbial diagnostics · Antiinfective treatment $\cdot$ Blood cultures systems zur vereinfachten Asservierung von Blutkulturen eine Maßnahme zur Reduktion von Kontaminationsmöglichkeiten installiert. Für die Blutentnahme wurde das VACUETTE ${ }^{\circledR}$-System um einen Blutkulturhalter (Greiner Bio-One $\mathrm{GmbH}$, Frickenhausen, Deutschland, Artikel-Nr. 450181) ergänzt. Die fachgerechte Anwendung des Blutentnahmeadapters für die Blutkulturen wurden im Rahmen der Schulungsmaßnahmen vermittelt.

\section{Erfassung von Qualitäts- und Quantitätsindikatoren}

Um die Wirksamkeit der Maßnahmen $\mathrm{zu}$ evaluieren, wurden routinemäßig bestimmte Qualitäts- und Quantitätsindikatoren in der nachfolgenden Vorund-nachher-Untersuchung gegenübergestellt. Folgende Prozessindikatoren wurden im Rahmen der vorbeschriebenen Qualitätssicherungsmaßnahmen erfasst:
- Rate abgenommener Blutkulturen/1000 Patienten;

- Anzahl positiver Blutkulturen;

- Anzahl mutmaßlicher Kontamination in den positiven Blutkulturen (retrospektive Ermittlung bei Nachweis von Hautkeimen - koagulasenegative Staphylokokken, Cutibacterium acnes, coryneforme Bakterien - anhand der beim jeweiligen Patienten gestellten Diagnose). 


\section{Originalien}

Tab. 1 Ergebnisse der retrospektiven Untersuchung vor und nach Entwicklung, Einführung und Training der SOP „Blutkulturen

Untersuchungszeitraum 11/17 bis 01/18

Vor Einführung der SOP „Blutkulturen"

Anzahl abgenommene Blutkulturen
Aerob
Anaerob
Nicht definiert
Anzahl Blutkulturen/1000 Fälle, $n$
Anzahl positiver Blutkulturen, $n$ (\%)
Davon typische Hautkeime, $n(\%)$
Davon plausibler Foci (It. Patientenakte, $n(\%)$

Typische Hautkeime ohne plausiblen Focus

(Kontaminationsrate, $n$ (\%)
Untersuchungszeitraum 11/2018 bis 01/2019

Nach Einführung der SOP „Blutkulturen“

2872

1430

1442

0

481

$394(13,7 \%)$

$129(32,7 \%)$

$25(19,4 \%), 6$ Pat.

4-mal Portinfekt

Einmal Demers-Katheter-Infekt

Einmal Gangrän bei AVK

$104(26,4 \%)$

SOP Standard Operating Procedure, $n$ Anzahl, Pat. Patienten, inf. infiziert, AVK Arterielle Verschlusskrankheit

\section{Punktprävalenzanalyse}

Zur Prüfung einer adäquaten Indikationsstellung zur Blutkulturdiagnostik wurde an jeweils einem Tag der 2 Studienzeiträume eine Punktprävalenzerfassung durchgeführt. Dabei wurde überprüft, in welchem Umfang bei den am jeweiligen Tag in der zentralen Notaufnahme behandelten Patienten mit Vorliegen entsprechender Symptome eine Blutkulturdiagnostik initiiert wurde, die den Kriterien der SOP Blutkulturdiagnostik entsprach.

\section{Zeitlicher Verlauf}

Im Zeitraum zwischen 01.09.2018 und 31.10.2018 erfolgten die Schulungsmaßnahmen und die Einführung der SOP Blutkulturdiagnostik. Am 01.11.2018 erfolgte der offizielle Start der Implementierung der SOP „Blutkulturen“ in der zentralen Notaufnahme. Als postinterventioneller Erfassungszeitraum wurde der Bereich vom 01.11.2018 bis 31.01.2019 gewählt. Als Vergleichszeitraum wurde der Vorjahreszeitraum vom 01.11.2017 bis 31.01.2018 gewählt, um den Einfluss einer Saisonalität (z. B. von Atemwegsinfektionen) zu reduzieren.

Die Punktprävalenzerfassung der korrekten Indikationsstellung zur Blutkulturdiagnostik erfolgte am 15.11.2017 und am 15.11.2018.

\section{Patientenkollektiv}

Zur Fallzahlplanung diente die Anzahl der nichttraumatologischen Patienten in der zentralen Notaufnahme in den genannten Zeiträumen. Bei den verarbeiteten Daten handelte es sich nicht um individuelle Patientendaten, sondern um Qualitätsindikatoren aus diagnostischen Ergebnissen, die auf die Gesamtzahl der behandelten Patienten in den Überprüfungszeiträumen bezogen wurden. Die Daten geben Aufschluss über die diagnostische Qualität im Gesamtkollektiv der behandelten Patienten. Zum Zweck der Qualitätskontrolle erfolgte eine primäre Erfassung der Daten ohne eine individuelle Aufklärung oder Einwilligung. Die errechneten Indikatoren lassen keinen Rückschluss auf individuelle Befunde zu. Es existierte kein Zuordnungsschlüssel, da eine Zuordnung technisch aus den ermittelten Qualitätsindikatoren nicht möglich war. Die Vorgaben der Datenschutzgrundverordnung (DSGVO) wurden eingehalten.

\section{Statistische Analyse}

Zur statistischen Auswertung wurden definierte Merkmale verglichen. Die Angaben der Daten erfolgten als Absolutzahl oder als prozentualer Anteil. Unterschiede zwischen den Patientenkollektiven wurde mittels $\chi^{2}$-Test analysiert. Eine Fehlerwahrscheinlichkeit von $p<0,05$ wurde als statistisch signifikant gewertet.

\section{Ergebnisse}

Die Schulungsmaßnahme erreichte 48 von 52 pflegerischen Mitarbeitern (92\%) und 26 von 28 ärztlichen Mitarbeitern (93\%). Die Anzahl der abgenommenen Blutkulturen in dem jeweiligen Zeitraum $11 / 2017$ bis $01 / 2018$ und $11 / 2018$ bis $01 / 2019$ hat sich von 1757 auf 2872 erhöht (•Tab. 1). Die Anzahl der abgenommen Blutkulturen/1000 Fälle von 287 auf 481 hat sich nahezu verdoppelt (• Abb. 2). Die Anzahl positiver Blutkulturen sank im Untersuchungszeitraum von $18,6 \%$ (326) auf $13,7 \%$ (394, $p<0,05)$. Ebenfalls konnte der Anteil typischer Hauptkeime von 38,7\% (126) auf $31,7 \%$ (129) reduziert werden $(p<0,05)$. In der retrospektiven Auswertung der Patientenakten wurde festgestellt, dass bei einem Teil der Patienten die nachgewiesenen Hautkeime mutmaßlich doch Ursache der Infektkonstellation waren, so in $11,1 \%$ $(n=3)$ im Vergleichszeitraum und in $19,4 \%(n=6)$ des postinterventionellen Erfassungszeitraums ( $p<0,05$; - Tab. 1). Der Anteil typischer Hauptkeime ohne plausiblen Fokus als Marker einer mutmaßlichen Kontamination sank somit von $34,4 \%(n=112)$ auf $26,4 \%(n=104$, $p<0,05, \bullet$ Abb. 3$)$.

\section{Prävalenzuntersuchungen}

$\mathrm{Zu}$ den 2 definierten Zeitpunkten am 15.11.2017 und 15.11.2018 wurden 94 


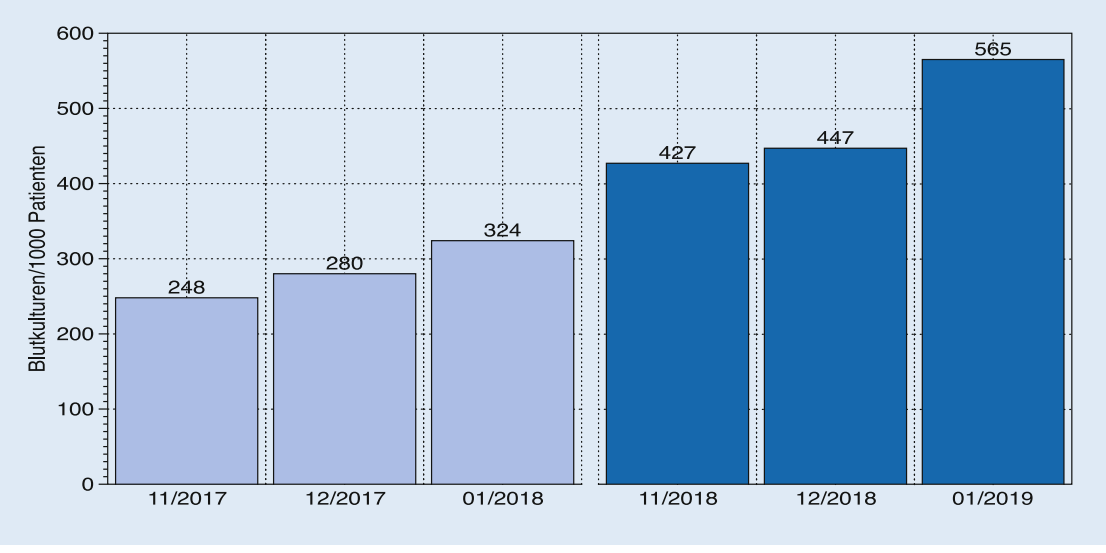

Abb. 2 ム Blutkulturen pro 1000 Patienten vor (hellblau) und nach (dunkelblau) Entwicklung, Training und Einführung der Standard Operating Procedure „Blutkulturen“ in der zentralen Notaufnahme

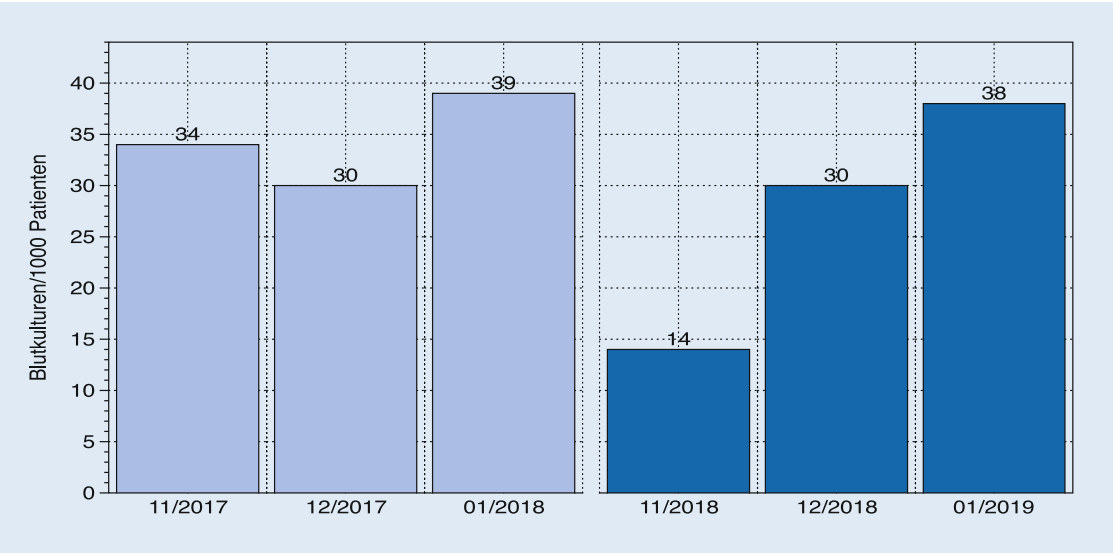

Abb. $3 \Delta$ Positive Blutkulturen pro 1000 Patienten mit Hautkeimen (kontaminierte Blutkulturen) vor (hellblau) und nach (dunkelblau) Entwicklung, Training und Einführung der Standard Operating Procedure „Blutkulturen“ in der Zentralen Notaufnahme

bzw. 105 Patienten in der zentralen Notaufnahme behandelt. Gemäß den Definitionen der SOP „Blutkulturen“ in der zentralen Notaufnahme ergab sich bei 7 Patienten im ersten Zeitraum und 5 Patienten im zweiten Zeitraum gemäß der SOP „Blutkulturen“ die Indikation zur Blutkulturdiagnostik. In allen Fällen und Situationen wurden Blutkulturen bei bestehender Indikation durch die Mitarbeiter abgenommen (Erfolgsrate: $100 \%)$. Die korrekte Anzahl abgenommener Blutkulturflaschen gemäß der Definition der SOP „Blutkultur“ in der zentralen Notaufnahme war jedoch im ersten Zeitraum mit $86 \%$ höher als im zweiten Zeitraum mit $60 \%$.
ABS-Teams helfen unter anderem, durch regelmäßige Visiten auf Stationen einen rationalen Umgang mit Antiinfektiva herbeizuführen. Ergänzend unterstützen ABS-Teams aber auch in der geeigneten Auswahl an mikrobieller Probengewinnung und im Umgang mit Blutkulturen als Diagnostikum im realen Stationsalltag [9]. Vor diesem Hintergrund wurde es als gemeinsame Zielsetzung des ABS-Teams und der Zentralen Notaufnahme des Universitätsklinikums Düsseldorf angesehen, die Quantität und Qualität der Blutkulturdiagnostik am eigenen Standort zu verbessern.

Mehrere Schritte sind dabei notwendig, um entsprechende Maßnahmen effektiv umzusetzen [3]: 1) Schulungen und Feedback (,education and feedback“), 2) die Etablierung von Bündeln/ Standard Operating Procedures („bundled approaches"), und 3) Technik und Material ("technique and equipment"). Entsprechend diesen Vorgaben wurden folgende Maßnahmen initialisiert: 1) Durch Schulungsmaßnahmen konnten über $90 \%$ der pflegerischen und ärztlichen Mitarbeiter der zentralen Notaufnahme geschult werden. 2) Dies erfolgte, nachdem interdisziplinär durch das ABSTeam und Mitarbeiterinnen und Mitarbeiter der zentralen Notaufnahme eine übersichtliche SOP „Blutkulturen“ entwickelt worden war. 3) Um auch hinsichtlich technischer und materieller Aspekte eine Optimierung herbeizuführen, wurde ein Blutentnahmeadapter eingeführt, der ein direktes Anschließen der Blutkulturflaschen an die Punktionskanüle ermöglicht und damit kontaminationsund verletzungsträchtige Arbeitsschritte wie ein Umfüllen des abgenommenen Bluts obsolet macht.

Die ausgeführten Maßnahmen hatten sowohl hinsichtlich der Qualitäts- als aus auch Quantitätsindikatoren positive Auswirkungen: Die Anzahl der durchgeführten Blutkulturen pro 1000 Fälle stieg nach Intervention um $86 \%$ von 287 auf 481 . Die Rate an Hautkeimen ohne plausiblen Fokus sank im ersten Monat nach Intervention von $34 \%$ auf $14 \%$, stieg aber im weiteren Verlauf auf das Ausgangsniveau wieder an. Dies verdeutlicht das nur transiente Wirken von Schulungs- 
maßnahmen, die in regelmäßigen $\mathrm{Ab}$ ständen wiederholt werden sollten. Der Anteil korrekt durchgeführter Indikationsstellungen konnte laut Punktprävalenzanalyse nicht weiter gesteigert werden, hier wurde in beiden Fällen eine Erfolgsrate von $100 \%$ erreicht.

Aufgrund der geringen Fallzahlen sind jedoch insbesondere die Ergebnisse der Punktprävalenzerfassung mit großer Vorsicht zu interpretieren und nur mit Zurückhaltung zu bewerten. Darüber hinaus muss beachtet werden, dass insbesondere die Anzahl an falschpositiven Befunden und damit an Kontaminationen im zweiten 3-monatigen Untersuchungsintervall wieder fast auf das Ausgangsniveau anstieg. Dies kann ein starker Hinweis darauf sein, dass Schulungsmaßnahmen hinsichtlich der Hygiene bei der Abnahme von Blutkulturen regelmäßig fortgesetzt werden müssen.

Besonders positiv könnte sich die Auswahl der zentralen Notaufnahme als Umsetzungslokalisation für qualitätssteigernde Maßnahmen bei der mikrobiellen Diagnostik aber vor allem daher erweisen, da regelmäßig eine Vielzahl an Rotationsassistenten aus verschiedenen Kliniken und Fachabteilungen hier tätig ist und diese die erlernten Informationen zur Blutkulturdiagnostik zu einem späteren Zeitpunkt in ihre eigenen Kliniken und Abteilungen transportieren können und somit als Multiplikatoren dienen.

\section{Limitationen}

In der vorliegenden retrospektiven Untersuchung wurden folgende Punkte nicht erfasst: der korrekte Zeitpunkt der Blutkulturdiagnostik (Abnahme der Blutkulturen vor oder nach Antibiotikaerstgabe) sowie die Korrektheit der Befüllvolumina der Blutkulturflaschen. Der Anteil der Wirksamkeit von Schulungsmaßnahmen und neuen Blutabnahmesystemen konnte aufgrund der Gleichzeitigkeit der Einführung nicht differenziert beziffert werden. Die geringe Diskrepanz der Anzahl aerober und anaerober Blutkulturflaschen ( $\bullet$ Tab. 1) deutet auf eine in Einzelfällen unvollständige Blutkulturabnahme hin. Möglicherweise lag diesen Fällen eine Dislokation der Punktionskanülen zugrunde, diese Annahme kann jedoch retrospektiv nicht überprüft werden.

Die pflegerische und ärztliche Besetzung in der zentralen Notaufnahme unterliegt einer hohen Fluktuation, einerseits durch Stellenwechsel, andererseits durch Rotationswechsel in der Weiterbildung. Vor diesem Hintergrund ist es sehr wahrscheinlich, dass sich in beiden retrospektiven Untersuchungszeiträumen die personellen Besetzungen unterschieden haben. Die Auswirkung dieser Personalfluktuation kann an dieser Stelle nicht bestimmt werden, die Einführung der SOP soll zukünftig diesen Einfluss aber reduzieren.

Die Folgeeffekte der Maßnahmen können quantitativ reduzierte und qualitativ bessere, weil für den korrekten Erreger angepasste, Antibiotikatherapien sein und somit positiven Einfluss auf Nebenwirkungen, Liegezeiten, Behandlungsergebnisse und Kosteneffektivität haben. Dies wurde aber in der vorliegenden Untersuchung nicht spezifisch erfasst.

\section{Fazit für die Praxis}

Eine in Zusammenarbeit zwischen ABSTeam und zentraler Notaufnahme entwickelte, geschulte und eingeführte SOP „Blutkulturen“ kann unter Verbesserung der materiellen Rahmenbedingungen (Blutentnahmeadapter) zur Optimierung der Blutkulturdiagnostik führen und sowohl die Quantität als auch die Qualität erhöhen.

\section{Korrespondenzadresse}

PD Dr. M. Bernhard, MHBA

Zentrale Notaufnahme, Universitätsklinikum Düsseldorf

Moorenstraße 5, 40225 Düsseldorf,

Deutschland

michael.bernhard@med.uni-duesseldorf.de

Danksagung. Die Autoren danken den Pflegenden und Ärzten der Zentralen Notaufnahme des Universitätsklinikums Düsseldorf für die engagierte Mitarbeiter bei der Einführung und täglichen Umsetzung der SOP „Blutkulturen“.

Funding. Open Access funding provided by Projekt DEAL.

\section{Einhaltung ethischer Richtlinien}

Interessenkonflikt. B.-E. O. Jensen erhielt Vortragshonorare von Janssen Cilag und Falk Foundation und Honorare für Beratertätigkeiten für Gilead und Thera Technologies, außerhalb der vorliegenden Arbeit. H. M. Orth, S. Al Agha, M. Kempe, C. Mackenzie, M. Michael und M. Bernhard geben an, dass kein Interessenkonflikt besteht.

Für diesen Beitrag wurden von den Autoren keine Studien an Menschen oder Tieren durchgeführt. Für die aufgeführten Studien gelten die jeweils dort angegebenen ethischen Richtlinien.

Open Access. Dieser Artikel wird unter der Creative Commons Namensnennung 4.0 International Lizenz veröffentlicht, welche die Nutzung, Vervielfältigung, Bearbeitung, Verbreitung und Wiedergabe in jeglichem Medium und Format erlaubt, sofern Sie den/die ursprünglichen Autor(en) und die Quelle ordnungsgemäß nennen, einen Link zur Creative Commons Lizenz beifügen und angeben, ob Änderungen vorgenommen wurden.

Die in diesem Artikel enthaltenen Bilder und sonstiges Drittmaterial unterliegen ebenfalls der genannten Creative Commons Lizenz, sofern sich aus der Abbildungslegende nichts anderes ergibt. Sofern das betreffende Material nicht unter der genannten Creative Commons Lizenz steht und die betreffende Handlung nicht nach gesetzlichen Vorschriften erlaubt ist, ist für die oben aufgeführten Weiterverwendungen des Materials die Einwilligung des jeweiligen Rechteinhabers einzuholen.

Weitere Details zur Lizenz entnehmen Sie bitte der Lizenzinformation auf http://creativecommons.org/ licenses/by/4.0/deed.de.

\section{Literatur}

1. Rhodes A, Evans LE, Alhazzani W et al (2017) Surviving sepsis campaign: international guidelines for management of sepsis and septic shock: 2016 . Crit Care Med 45:486-552

2. Levy MM, Evans LE, Rhodes A (2018) The surviving sepsis campaign bundle: 2018 update. Intensive Care Med 44:925-928

3. Bool M, Barton MJ, Zimmerman PA (2020) Blood culture contamination in the emergency department: an integrative review of strategies to prevent blood culture contamination. Australas Emerg Care. https://doi.org/10.1016/j.auec.2020. 02.004

4. Kai M, Miyamoto K, Akamatsu K, Tsujita A, Nishio M (2020) Effect of a bundle-approach intervention against contamination of blood culture in the emergency department. J Infect Chemother. https://doi.org/10.1016/j.jiac.2020.03.005

5. Macdonald SPJ, Williams JM, Shetty A et al (2017) Sepsis in the emergency department-part 1: definitions and outcomes. Emerg Med Australas 29:619-625

6. Shetty A, Macdonald SPJ, Keijzers G et al (2018) Sepsis in the emergency department-part 2: investigations and monitoring. Emerg Med Australas 30:4-12

7. Williams JM, Keijzers G, Macdonald SPJ et al (2018) Sepsis in the emergency department-part 3: treatment. Emerg Med Australas 30:144-151 
8. Richter SS, Beekmann SE, Croco JLet al (2002) Minimizing the workup of blood culture contaminants: implementation and evaluation of a laboratorybased algorithm. J Clin Microbiol 40:2437-2444

9. Tabah A, Bassetti M, Kollef MH et al (2020) Antimicrobial de-escalation in critically ill patients: a position statement from a task force of the European society of intensive care medicine (ESCIM) and European society of clinical microbiology and infection disease (ESCMID) critcially ill patients study group (ESGCIP). Intensive Care Med 46:245-265

\section{Buchempfehlungen in der AINS aus dem Springer-Verlag}

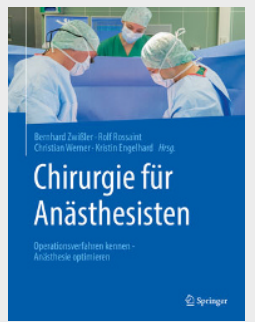

\section{Chirurgie für Anästhesisten}

Zwißler, B., Rossaint, R., Werner, C., Engelhard, K. (Hrsg.) | 1. Aufl. 2021, XVII, 765, Hardcover 129,99€, ISBN 978-3-662-53337-6

In diesem Buch beschreiben interdisziplinäre Autorenteams, bestehend aus Chirurgen und Anästhesisten, die gängigsten Operationsverfahren aus den chirurgischen Fachgebieten und deren Bedeutung für das anästhesiologische Management. Das Werk wendet sich an alle Anästhesisten in Aus-und Weiterbildung sowie Fachärzte für Anästhesie, die beim Blick über das OP-Tuch noch besser verstehen möchten, was der Eingriff für ihren Patienten bedeutet - präoperativ, intraoperativ und postoperativ.

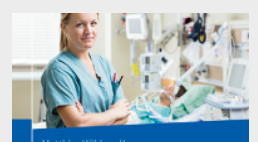

Komplikationen

in der Intensiv-

medizin

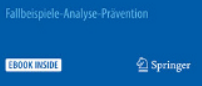

\section{Komplikationen in der Intensivmedizin}

Hübler, Matthias (Hrsg.) | 1. Aufl. 2019, XX, 412, Softcover 44,99€, eBook inside, ISBN 978-3-662-58307-4

Komplikationen oder Zwischenfälle sind häufig eine Kombination menschlicher, organisatorischer und technischer Fehler. Das vorliegende Werk zeigt 24 Fallbeispiele aus der Intensivmedizin, bei denen es zu Komplikationen oder Beinahezwischenfällen gekommen ist. Mit „Komplikationen in der Intensivmedizin“ erscheint nach „Komplikationen in der Anästhesie" das zweite Werk zum Themenkreis in gewohnt spannender, kurzweiliger und lehrreicher Art und Weise.

\section{Neurologische Beatmungsmedizin}

Groß, Martin (Hrsg.) | 1. Aufl. 2020, XIX, 544, Hardcover 79,99€, ISBN 9783-662-59013-3

Neurologische

Beatmungsmedizin

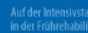

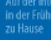

QSpringer

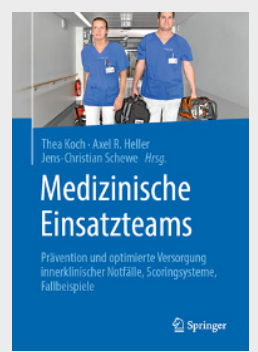

\section{Medizinische Einsatzteams}

Koch, Thea, Heller, Axel R., Schewe, Jens-Christian (Hrsg.) | 1. Aufl. 2019, XX, 245, Softcover 39,99€, ISBN 978-3-662-58293-0

Das Werk liefert praxisbezogenes Expertenwissen rund um die Implementierung und Bedeutung von medizinischen Einsatzteams in Krankenhäusern. Besonders praxisrelevant: Fallbeispiele häufiger Notfallsituationen, systematisch dargestellt nach Symptomen, Alarmierungsgrund, Eintreffen des MET, Diagnostik, Maßnahmen, Verlauf, Outcome sowie Besonderheiten und Auswertung.

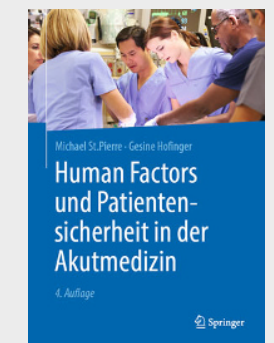

Human Factors und Patientensicherheit in der Akutmedizin St.Pierre, Michael, Hofinger, Gesine (Hrsg.) | 4. Aufl. 2020, XII, 394, Hardcover 79,99€, ISBN 978-3-662-60484-7

Menschliches Entscheiden und Handeln wird von zahlreichen Faktoren beeinflusst, die eine sichere Versorgung gefährden. Welche „Human Factors" einen maßgeblichen Einfluss haben, beschreibt das vorliegende Werk praxisnah und anhand zahlreicher Fallbeispiele. Die 4. Auflage erscheint komplett aktualisiert und erweitert. 